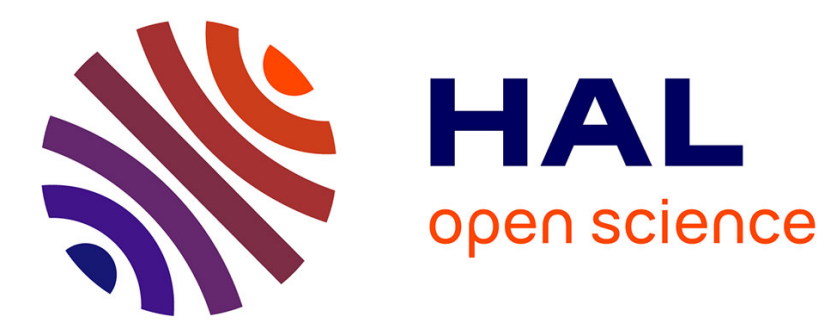

\title{
Spin injection in silicon at zero magnetic field
}

\author{
L. Grenet, M. Jamet, P. Noe, V. Calvo, J.-M. Hartmann, L. E. Nistor, B. \\ Rodmacq, S. Auffret, P. Warin, Y. Samson
}

\section{To cite this version:}

L. Grenet, M. Jamet, P. Noe, V. Calvo, J.-M. Hartmann, et al.. Spin injection in silicon at zero magnetic field. Applied Physics Letters, 2009, 94 (3), pp.032502. 10.1063/1.3064135 . cea-01055870

\section{HAL Id: cea-01055870 https://hal-cea.archives-ouvertes.fr/cea-01055870}

Submitted on 18 Aug 2014

HAL is a multi-disciplinary open access archive for the deposit and dissemination of scientific research documents, whether they are published or not. The documents may come from teaching and research institutions in France or abroad, or from public or private research centers.
L'archive ouverte pluridisciplinaire HAL, est destinée au dépôt et à la diffusion de documents scientifiques de niveau recherche, publiés ou non, émanant des établissements d'enseignement et de recherche français ou étrangers, des laboratoires publics ou privés. 


\section{AIP Apleted Pyysics}

\section{Spin injection in silicon at zero magnetic field}

L. Grenet, M. Jamet, P. Noé, V. Calvo, J.-M. Hartmann, L. E. Nistor, B. Rodmacq, S. Auffret, P. Warin, and Y. Samson

Citation: Applied Physics Letters 94, 032502 (2009); doi: 10.1063/1.3064135

View online: http://dx.doi.org/10.1063/1.3064135

View Table of Contents: http://scitation.aip.org/content/aip/journal/apl/94/3?ver=pdfcov

Published by the AIP Publishing

\section{Articles you may be interested in}

Electrical spin injection into $\mathrm{p}$-doped quantum dots through a tunnel barrier

Appl. Phys. Lett. 90, 081111 (2007); 10.1063/1.2709889

Spin-polarized light-emitting diode using metal/insulator/semiconductor structures

Appl. Phys. Lett. 81, 694 (2002); 10.1063/1.1496493

Interface characterization and thermal stability of $\mathrm{Co} / \mathrm{Al}-\mathrm{O} / \mathrm{CoFe}$ spin-dependent tunnel junctions

J. Appl. Phys. 91, 7475 (2002); 10.1063/1.1452228

Magnetoresistance of spin-dependent tunnel junctions with composite electrodes

J. Appl. Phys. 90, 6222 (2001); 10.1063/1.1419259

Spin-polarized tunneling in a half-metallic ferromagnet

J. Appl. Phys. 86, 6239 (1999); 10.1063/1.371678

\section{Asylum Research Atomic Force Microscopes}

Unmatched Performance, Versatility and Support

Modulus of Polymers

\& Advanced Materials

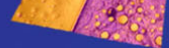

Coating Uniformity

\& Roughness

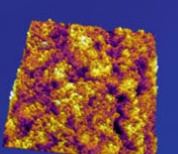

Nanoscale Conductivity \& Permittivity Mapping

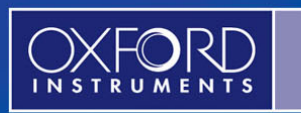

The Business of Science

\section{Piezoelectrics}

\& Ferroelectrics
+1 (805) 696-6466 sales@AsylumResearch.com www.AsylumResearch.com 


\title{
Spin injection in silicon at zero magnetic field
}

\author{
L. Grenet, ${ }^{1,2, a)}$ M. Jamet, ${ }^{1,2}$ P. Noé, ${ }^{1,2}$ V. Calvo, ${ }^{1,2}$ J.-M. Hartmann, ${ }^{3}$ L. E. Nistor, ${ }^{4}$ \\ B. Rodmacq, ${ }^{4}$ S. Auffret, ${ }^{4}$ P. Warin, ${ }^{1,2}$ and Y. Samson ${ }^{1,2}$ \\ ${ }^{1}$ CEA, INAC, SP2M, 17 rue des martyrs, F-38054 Grenoble, France \\ ${ }^{2}$ Université Joseph Fourier, SP2M, F-38041 Grenoble, France \\ ${ }^{3}$ CEA, LETI, F-38054 Grenoble, France \\ ${ }^{4}$ SPINTEC, CEA/CNRS/UJF/GINP INAC, 38054 Grenoble Cedex, France
}

(Received 13 November 2008; accepted 12 December 2008; published online 20 January 2009)

\begin{abstract}
In this letter, we show efficient electrical spin injection into a SiGe based $p$-i- $n$ light emitting diode from the remanent state of a perpendicularly magnetized ferromagnetic contact. Electron spin injection is carried out through an alumina tunnel barrier from a Co/Pt thin film exhibiting a strong out-of-plane anisotropy. The electron spin polarization is then analyzed through the circular polarization of emitted light. All the light polarization measurements are performed without an external applied magnetic field, i.e., in remanent magnetic states. The light polarization as a function of the magnetic field closely traces the out-of-plane magnetization of the $\mathrm{Co} / \mathrm{Pt}$ injector. We could achieve a circular polarization degree of the emitted light of $3 \%$ at $5 \mathrm{~K}$. Moreover this light polarization remains almost constant at least up to $200 \mathrm{~K}$. (C) 2009 American Institute of Physics. [DOI: $10.1063 / 1.3064135]$
\end{abstract}

Spintronics aims at manipulating both charge and spin of carriers and has been growing tremendously fast for the past 10 years. ${ }^{1}$ Indeed it has been predicted as an alternative solution to the conventional complementary metal oxide semiconductor technology that will reach physical limitations in the near future. ${ }^{2}$ This subject relies on efficient spin injection into semiconductor heterostructures. Moreover for integration purposes, spin injection should be carried out electrically. So far, electrical spin injection has been widely demonstrated in III-V semiconductors using both ferromagnetic metals and magnetic semiconductors. The spin polarization of injected carriers is then given by the degree of circular polarization of the light emitted by a GaAs based spin-light emitting diode (LED) (Ref. 3) through dipolar selection rules. The most spectacular spin injection and optical detection were performed from BeMnZnSe, ${ }^{4} \mathrm{Fe},{ }^{5}$ or $\mathrm{CoFe}$ (Ref. 6) injectors. Only recently, spin injection in silicon has been achieved using ferromagnetic metals. Spin polarized hot electrons were injected in a silicon wafer, and spin detection was achieved by electrical means using two ferromagnetic electrodes in a giant magnetoresistancelike geometry. ${ }^{7}$ In parallel, Jonker et al. ${ }^{8}$ demonstrated spin injection in the conduction band of silicon by measuring the circular polarization of a Si $p-i-n$ LED. It should be stressed that silicon appears as the most promising candidate for spintronic circuitry and quantum computing systems since very long diffusion lengths and coherence times are predicted in this material, owing to its low atomic mass (low spin-orbit coupling) and inversion symmetry of the crystal itself. Moreover the dominant naturally occurring isotope has no nuclear spin, ruling out spin relaxation due to hyperfine interactions. ${ }^{9,10}$

In this letter, we demonstrate spin injection into silicon using the remanent state of a perpendicularly magnetized ferromagnetic semitransparent contact. The electron spin polarization is detected optically using a Si based spin-LED. The

\footnotetext{
${ }^{\text {a) }}$ Author to whom correspondence should be addressed. Electronic mail: louis.grenet@cea.fr.
}

active region is a fully strained $\mathrm{Si}_{0.7} \mathrm{Ge}_{0.3}$ quantum well (QW). Compared to pure silicon spin-LEDs, the presence of germanium enhances the spin-orbit coupling, which is required to observe circularly polarized light from the recombination of spin polarized injected electrons with holes. Spin injection from the $\mathrm{Co} / \mathrm{Pt}$ ferromagnetic metal is carried out in the tunneling regime through an alumina barrier to overcome the conductivity mismatch obstacle. ${ }^{11}$ Dipolar selection rules in spin-LEDs require perpendicular magnetization. In most experiments on spin injection, ferromagnetic films exhibit in-plane magnetization due to shape anisotropy. Hence strong external magnetic fields ( $2.2 \mathrm{~T}$ in the case of iron) are applied to saturate the film magnetization perpendicular to the QW plane. This may be the cause of many spurious effects such as magnetic circular dichroism (MCD) in the experimental setup itself or in the spin-LED heterostructure. Moreover the Zeeman effect in the active region artificially increases spin lifetimes. Very few experiments have been carried out in remanent magnetic states (i.e., with no applied field) into GaAs based spin-LEDs. Adelmann et al. ${ }^{12}$ reported a remanent spin injection of about $5 \%$ at $2 \mathrm{~K}$ using the $\delta$-MnGa Schottky contact. Gerhardt et al. ${ }^{13}$ demonstrated remanent spin injection using $\mathrm{Fe} / \mathrm{Tb}$ multilayers with a circular polarization of $0.75 \%$ at $90 \mathrm{~K}$. Finally Sinsarp et al. ${ }^{14}$ used a FePt/MgO spin injector and obtained a lower estimate of spin injection of $1.5 \%$ at room temperature. In this work, the $\mathrm{Co} / \mathrm{Pt}$ ferromagnetic film exhibits strong perpendicular magnetic anisotropy, allowing us to make spin injection in different remanent states.

We used the reduced pressure chemical vapor deposition in order to grow at 26.6 mbar the following stack on a slightly $p$-type doped $\mathrm{Si}(001)$ substrate (resistivity in the $7-10 \Omega \mathrm{cm}$ range): boron-doped $\mathrm{Si}\left(10^{19} \mathrm{~cm}^{-3}\right) 500 \mathrm{~nm} /$ intrinsic $\mathrm{Si}(50 \mathrm{~nm}) / \mathrm{SiGe} 30 \%(10 \mathrm{~nm}) /$ intrinsic $\mathrm{Si}(50 \mathrm{~nm}) /$ phosphorous-doped $\mathrm{Si}\left(10^{18} \mathrm{~cm}^{-3}\right) 50 \mathrm{~nm}$. The growth temperature was around $650{ }^{\circ} \mathrm{C}$ in order to avoid any significant surface roughening of the $\mathrm{Si}_{0.7} \mathrm{Ge}_{0.3}$ layer during its capping with $\mathrm{Si}$. The gaseous precursors used were silane 


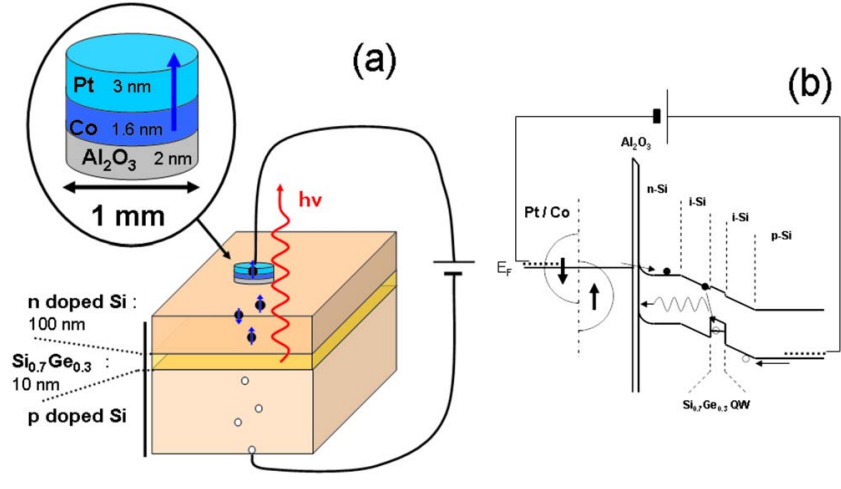

FIG. 1. (Color online) (a) Schematic device structure of the SiGe spin-LED with the $\mathrm{Co} / \mathrm{Pt}$ ferromagnetic top electrode and (b) associated simplified band diagram under bias in the injection regime.

(for the Si layers) and dichlorosilane+diluted germane (for the SiGe layer). For the boron (phosphorus) doping, we have used diborane (phosphine). High purity hydrogen with a flow of a few tens of SLM (SLM denotes standard liters per minute) was used as the carrier gas. This SiGe based spinLED is subsequently protected with a $2 \mathrm{~nm}$ thick thermal $\mathrm{SiO}_{2}$ layer for air transfer. Prior to the deposition of the tunnel barrier and ferromagnetic layer, this protective layer is etched using hydrofluoric acid and rinsed in de-ionized water. A $2 \mathrm{~nm}$ thick alumina tunnel barrier is then deposited at room temperature using radio frequency magnetron sputtering. The ferromagnetic layer is grown by direct current magnetron sputtering at room temperature through a mechanical mask defining $900 \mu \mathrm{m}$ wide mesas. It consists of a $1.6 \mathrm{~nm}$ of Co covered with $3 \mathrm{~nm}$ of Pt. The as-grown ferromagnetic layer exhibits an in-plane magnetization. After annealing the whole heterostructure at $420{ }^{\circ} \mathrm{C}$ for $1 \mathrm{~h}$ and $30 \mathrm{~min}$ the $\mathrm{Co}$ layer magnetization points out of plane. The whole structure as well as the band structure ${ }^{15}$ is sketched in Fig. 1.

Superconducting quantum interference device (SQUID) measurements with the applied field perpendicular to the film plane are displayed in Fig. 2. In Fig. 2(a) are hysteresis loops recorded at different temperatures. As shown in Fig. 2(b), the coercive field strongly decreases with temperature by $88.3 \%$. However low coercive fields at room temperature are required to switch easily up and down the magnetization of the spin injector in future spintronic applications. In the inset of Fig. 2(b), we have plotted the temperature dependence of the saturation magnetization. The saturation magnetization is the one of bulk cobalt at low temperature. It however decreases by $30.5 \%$ when temperature increases from 5 to $400 \mathrm{~K}$. This temperature dependence follows a Bloch law and we could
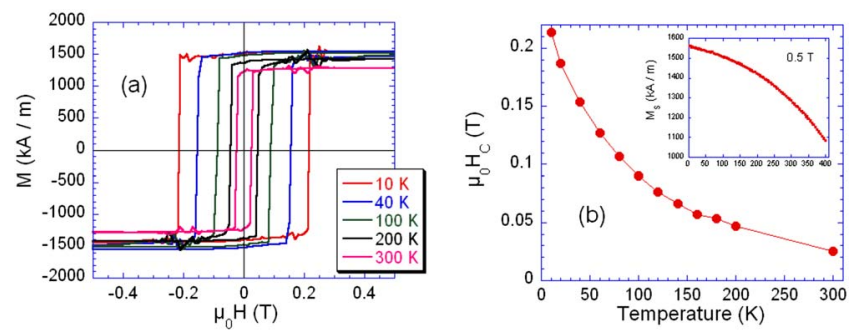

FIG. 2. (Color online) (a) Hysteresis loops of the Co/Pt ferromagnetic contact recorded at different temperatures. (b) Temperature dependence of the coercive field. Inset: temperature dependence of the saturation magnetizatien under $0.5 \mathrm{~T}$.
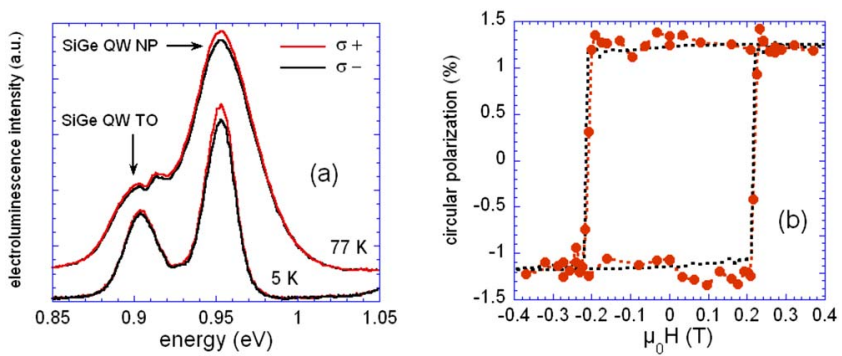

FIG. 3. (Color online) (a) EL spectra recorded at 5 and $77 \mathrm{~K}$ for a saturated remanent state of the ferromagnetic $\mathrm{Co} / \mathrm{Pt}$ contact. The applied voltage and current are respectively around $10 \mathrm{~V}$ and $10 \mathrm{~mA}$. The NP peak is clearly circularly polarized, indicating an efficient spin injection into the silicon top layer. The TO replica is also slightly circularly polarized. (b) EL circular polarization recorded at the maximum of the NP line for different remanent states of the $\mathrm{Co} / \mathrm{Pt}$ layer (red dots). The black dotted line corresponds to the SQUID measurement performed at the same temperature.

roughly estimate a Curie temperature of $900 \mathrm{~K}$, which is much lower than bulk cobalt $\left(T_{C}=1400 \mathrm{~K}\right)$. This may be due to dimensionality effects or more probably to $\mathrm{Co}-\mathrm{Pt}$ intermixing during sample annealing. Since the coercive field is correlated with the magnetization, its sharp decrease with temperature can be explained by the magnetization temperature evolution. However, we could not find a simple power law for $H_{C}$ versus $M_{S}$.

We performed both photoluminescence (not shown) and electroluminescence (EL) measurements on the SiGe spinLED at various temperatures using a cooled InGaAs photomultiplier tube. The EL was excited with square electrical pulses at low frequency $(63.7 \mathrm{~Hz})$ with a $1: 1$ duty cycle, and the signal was recorded using a lock-in technique. The circular polarization $P=\left(I_{\sigma+}-I_{\sigma-}\right) /\left(I_{\sigma+}+I_{\sigma-}\right)$ is determined using a rotatable quarter waveplate and a fixed linear polarizer placed in front of the detector. In Fig. 3(a) are reported EL spectra recorded at 5 and $77 \mathrm{~K}$ for a saturated remanent state of the ferromagnetic contact. In addition to the silicon EL (not shown), the spectra are dominated by two main features at $\sim 950 \mathrm{meV}$ corresponding to the no phonon (NP) recombination line and $\sim 900 \mathrm{meV}$ corresponding to its TO phonon replica. ${ }^{15}$ Both lines are clearly circularly polarized as a consequence of momentum transfer between the spin polarized injected electrons and the emitted photons. In the following, we only consider the highest circular polarization of the NP line. We could reach a maximum of $P=3 \%$ at $5 \mathrm{~K}$. Moreover this circular polarization remains almost constant up to 200 $\mathrm{K}$. Above this temperature, the EL signal from the SiGe QW becomes too weak to measure the circular polarization. In order to rule out any extrinsic effects, we performed EL measurements using a gold semitransparent contact and inserting in the optical path a thin Si plate on which we deposited the $\mathrm{Co} / \mathrm{Pt}$ ferromagnetic layer. Hence we could confirm that $\mathrm{MCD}$ in the $\mathrm{Co} / \mathrm{Pt}$ layer in a saturated remanent state is negligible. Moreover, Zeeman effect in the $\mathrm{Si}_{0.7} \mathrm{Ge}_{0.3} \mathrm{QW}$ could be ruled out since the stray field from the saturated $\mathrm{Co} / \mathrm{Pt}$ contact is negligible as pointed out by Gerhardt et al. ${ }^{13}$

In Fig. 3(b), we have plotted the EL circular polarization for different magnetic remanent states of the ferromagnetic contact. In this experiment, EL measurements are performed without applied magnetic field. The reported magnetic field in the graph corresponds to the magnetic field required to reach a given remanent state of the magnetization of the Co/Pt layer. The circular polarization exactly traces the 
SQUID measurements, indicating that the spin orientation of the injected electrons that radiatively recombine in the $\mathrm{SiGe}$ QW reflects that of the electron spin orientation in the $\mathrm{Co} / \mathrm{Pt}$ film. The measured circular polarization corresponds to a lower bound of the actual spin injection in silicon. Indeed spin relaxation (with a characteristic time $\tau_{s f}$ ) may take place during the transit time of electrons in the silicon top layer as well as during the radiative lifetime $\left(\tau_{r}\right)$ in the SiGe QW. Neglecting spin relaxation in the silicon top layer, the actual electron spin polarization achieved in silicon is given by $P\left(1+\tau_{r} / \tau_{s f}\right)$, where the ratio $\tau_{r} / \tau_{s f}$ strongly depends on temperature and the exact electronic structure of the QW. We estimate the total lifetime in the QW; it is tens of nanoseconds, which is very short. However we have no clear estimate of the spin relaxation time $\left(\tau_{s f}\right)$ in the SiGe QW. The determination of $\tau_{s f}$ requires time-resolved spin dynamics measurements. We can only mention electron spin resonance measurements performed by Jantsch et al. ${ }^{10}$ on $\mathrm{Si}_{1-x} \mathrm{Ge}_{x}(x$ $<0.1)$ QWs grown pseudomorphically between $\mathrm{Si}_{1-y} \mathrm{Ge}_{y}(y$ $>0.2$ ) barriers giving $\tau_{s f} \approx 1 \mu \mathrm{s}$. In our case, with higher Ge content and strain, we believe that spin relaxation time should be shorter, although we cannot give a lower bound. Further experiments are thus needed to estimate the actual spin injection efficiency into silicon.

In summary, we have shown tunneling spin injection into silicon from the remanent state of a ferromagnetic $\mathrm{Co} / \mathrm{Pt}$ contact. A maximum circular polarization of $3 \%$ could be achieved at $5 \mathrm{~K}$ and remains almost constant up to $200 \mathrm{~K}$. Moreover spin injection was performed for different magnetic remanent states of the ferromagnetic layer, and the associated circular polarization exactly traces the SQUID mea- surements as expected for spin polarized electrons injected from the $\mathrm{Co} / \mathrm{Pt}$ contact.

The authors would like to thank L. Notin, A. Brenac, and D. Leroy for their technical assistance with the sample growth.

${ }^{1}$ S. A. Wolf, D. D. Awschalom, R. A. Buhrman, J. M. Daughton, S. von Molnar, M. L. Roukes, A. Y. Chtchelkanova, and D. M. Treger, Science 294, 1488 (2001).

${ }^{2}$ I. Zutic, J. Fabian, and S. Das Sarma, Rev. Mod. Phys. 76, 323 (2004).

${ }^{3}$ A. G. Aranov and G. E. Pikus, Sov. Phys. Semicond. 10, 698 (1976).

${ }^{4}$ R. Fiederling, M. Keim, G. Reuscher, W. Ossau, G. Schmidt, A. Waag, and L. W. Molenkamp, Nature (London) 402, 787 (1999).

${ }^{5}$ O. M. J. van't Erve, G. Kioseoglou, A. T. Hanbicki, C. H. Li, B. T. Jonker, R. Mallory, M. Yasar, and A. Petrou, Appl. Phys. Lett. 84, 4334 (2004).

${ }^{6}$ X. Jiang, R. Wang, R. M. Shelby, R. M. Macfarlane, S. R. Bank, J. S. Harris, and S. S. P. Parkin, Phys. Rev. Lett. 94, 056601 (2005).

${ }^{7}$ I. Appelbaum, B. Huang, and D. J. Monsma, Nature (London) 447, 295 (2007).

${ }^{8}$ B. T. Jonker, G. Kioseoglou, A. T. Hanbicki, C. H. Li, and P. E. Thompson, Nat. Phys. 3, 542 (2007).

${ }^{9}$ A. M. Tyryshkin, S. A. Lyon, W. Jantsch, and F. A. Schäffler, Phys. Rev. Lett. 94, 126802 (2005).

${ }^{10}$ W. Jantsch, H. Malissa, Z. Wilamowski, H. Lichtenberger, G. Chen, F. Schaffler, and G. Bauer, J. Supercond. Novel Magn. 18, 145 (2005).

${ }^{11}$ E. Rashba, Phys. Rev. B 62, R16267 (2000).

${ }^{12}$ C. Adelmann, J. L. Hilton, B. D. Schultz, S. McKernan, C. J. Palmstrom, X. Lou, H.-S. Chiang, and P. A. Crowell, Appl. Phys. Lett. 89, 112511 (2006).

${ }^{13}$ N. C. Gerhardt, S. Hovel, C. Brenner, M. R. Hofmann, F.-Y. Lo, D. Reuter, A. D. Wieck, E. Schuster, W. Keune, S. Halm, G. Bacher, and K. Westerholt, J. Appl. Phys. 99, 073907 (2006).

${ }^{14}$ A. Sinsarp, T. Manago, F. Takano, and H. Akinaga, Jpn. J. Appl. Phys., Part 2 46, L4 (2006).

${ }^{15}$ J. Weber and M. I. Alonso, Phys. Rev. B 40, 5683 (1989). 